\title{
Article \\ Host-Specific Parasites Reveal the History and Biogeographical Contacts of Their Hosts: The Monogenea of Nearctic Cyprinoid Fishes
}

\author{
Andrea Šimková ${ }^{1, * \mathbb{D}}$, Eva Řehulková $^{1}$, Anindo Choudhury ${ }^{2}$ and Mária Seifertová ${ }^{1}$ \\ 1 Department of Botany and Zoology, Faculty of Science, Masaryk University, Kotlářská 2, \\ 61137 Brno, Czech Republic; evar@sci.muni.cz (E.Ǩ.); 108415@mail.muni.cz (M.S.) \\ 2 Division of Natural Sciences, St. Norbert College, De Pere, WI 54115, USA; anindo.choudhury@snc.edu \\ * Correspondence: simkova@sci.muni.cz
}

check for updates

Citation: Šimková, A.; Řehulková, E.; Choudhury, A.; Seifertová, M. Host-Specific Parasites Reveal the History and Biogeographical Contacts of Their Hosts: The Monogenea of Nearctic Cyprinoid Fishes. Biology 2022, 11, 229. https:// doi.org/10.3390/biology11020229

Academic Editor: Oswaldo Palenzuela

Received: 8 December 2021 Accepted: 25 January 2022 Published: 31 January 2022

Publisher's Note: MDPI stays neutral with regard to jurisdictional claims in published maps and institutional affiliations.

Copyright: (C) 2022 by the authors. Licensee MDPI, Basel, Switzerland. This article is an open access article distributed under the terms and conditions of the Creative Commons Attribution (CC BY) license (https:// creativecommons.org/licenses/by/ $4.0 /)$.
Simple Summary: Parasites exhibiting close associations with their hosts may represent a useful tool when investigating historical biogeography, especially in the case of hosts associated with a once contiguous landmass. Host-specific gill parasites (Monogenea) were applied as a supplementary tool to reveal the historical biogeographical contacts between freshwater fish from North America and Europe and their contemporary contacts in North America. Cyprinoidei is the most speciesrich lineage of cypriniform fish with Leuciscidae exhibiting a Holarctic distribution. Monogenean parasites of the genus Dactylogyrus are mostly restricted to this freshwater fish group, and the high species diversity of Dactylogyrus follows the high diversity of their cyprinoid fish hosts. Using a phylogenetic approach, two Nearctic clades of Dactylogyrus spp. with different origins were revealed indicating two different historical routes of cyprinoid dispersion to the North American continent. Our study showed that the historical contacts between European and North American leuciscids were accompanied by the host switching of gill monogeneans. The phylogenetic relationships among North American Dactylogyrus spp. indicated numerous colonizations of cypriniform fish resulting from ancient paleogeographic events and contemporary drainage reorganization, thereby, facilitating contacts among phylogenetically distant fish species.

Abstract: Host-specific parasites exhibit close co-evolutionary associations with their hosts. In the case of fragmented/disjunct host distribution, host-specific parasites may reflect the biogeographical history of regions and/or the role played by contacts of hosts. The present study was focused on Dactylogyrus (Monogenea) species almost exclusively parasitizing cyprinoid fishes. We investigated the phylogenetic relationships between Dactylogyrus parasites of Nearctic cyprinoids (Leuciscidae) and Dactylogyrus parasites of Palearctic cyprinoids and used Dactylogyrus phylogeny to explore the biogeography of fish hosts in Europe and North America. Phylogenetic analyses revealed that two Nearctic clades of Dactylogyrus spp. have different origins. Historical contacts between European and North American leuciscids were accompanied by the host switching of Dactylogyrus species. In the Nearctic region, Dactylogyrus parasites also colonized non-leuciscid fishes. Dactylogyrus spp. of three Nearctic leuciscid clades were included in the phylogenetic reconstruction; only Dactylogyrus spp. of the Plagopterinae had a common origin. Dactylogyrus species did not reflect the phylogenetic relationships among leuciscid clades, suggesting that past co-diversification was overshadowed by colonization events mediated by paleogeographic and climatological changes and extensive drainage reorganization. Host-specific monogeneans serve as a supplementary tool to reveal the historical biogeographical contacts between freshwater fish from the North America and Europe and also contemporary contacts of leuciscids in North America.

Keywords: host-parasite associations; host-specific parasites; monogenea; fish; cyproniforms; phylogeny; biogeography; Nearctic area 


\section{Introduction}

Parasites are considered useful indicators of contemporary and historical ecology and biogeography on varying temporal and spatial scales [1,2]. They reveal processes involved in diversification and the formation of ecosystems and provide insights about the history and structure of faunal associations in evolutionary and ecological time $[1,3,4]$. The geographical distribution of parasites is limited by the distribution of their hosts (including historical and contemporary constraints on the host) and is closely associated with host dispersal capabilities. This phenomenon may be particularly evident in parasites with direct life cycles and exhibiting narrow host specificity (i.e., a parasite species is restricted to a given host species or a close range of phylogenetically-related host species).

Monogeneans are parasitic flatworms (Plathyhelminthes) with monoxenous life cycles (involving a single host), mostly infecting the gills and fins of fish. They are highly diverse in terms of species richness [5], morphology (various forms of the sclerotized parts of attachment and reproductive organs) and ecology (host and microhabitat specificities) [6-10]. Due to the close associations of monogeneans with their fish hosts, monogeneans represent a useful tool when investigating the historical biogeography of freshwater fish faunas with a fragmented/disjunct distribution, especially those that are associated with a once-contiguous landmass [11-15].

Among monogeneans, the genus Dactylogyrus is the most speciose, with the majority of species infecting freshwater fish of Cyprinoidei (formerly Cyprinidae, see [16]). The high species diversity of Dactylogyrus is closely linked to the high diversity of their cyprinoid fish hosts; however, a few non-cyprinoid hosts have also been documented [17]. Host switching (= host shifting) and parasite duplication (intrahost speciation) were revealed as the main processes of Dactylogyrus diversification in European cyprinoids $[13,18]$.

In addition, the rapid adaptive radiation of Dactylogyrus in geographically isolated regions was shown to be closely associated with the diversification of their endemic cyprinoid hosts in the peri-Mediterranean area [14]. Generally, Dactylogyrus species have been recognized to exhibit high host specificity; a large proportion of Dactylogyrus species are either strict specialists (i.e., a parasite species is specific to a single host species) or intermediate specialists (i.e., a parasite species is specific to a limited range of congeneric host species) [10]. However, even among Dactylogyrus, some species are able to infect a wide range of phylogenetically distant and even geographically isolated host species $[10,12,19,20]$.

Cyprinoidei is the most species-rich lineage of Cypriniformes and is currently represented by 12 families with Cyprinidae and Leuciscidae being the most diverse and widely distributed [21]. Leuciscidae (alternatively considered as Leuciscinae within Cyprinidae, see [21]) are distributed in Eurasia and North America (i.e., they exhibit a Holarctic distribution), whereas Cyprinidae (alternatively considered as Cyprininae within Cyprinidae) are found in Europe, Asia and Africa i.e., they are not native to the Nearctic region.

Inferring from a mitogenome phylogeny, Imoto et al. [22] proposed that Leuciscidae originated in Cretaceous Europe and diverged into two phyletic groups, leuciscins and phoxinins, the latter possibly originating later in North America. However, Schönhuth et al. [21], using multiple mitochondrial and nuclear genes to infer the phylogenetic relationships within Leuciscidae, revealed the complex evolutionary history of this widespread fish group. Their study suggested multiple connections and dispersal events between Palearctic and Nearctic regions and multiple shifts of leuciscids between pelagic and benthic habitats.

The phylogeny of Dactylogyrus parasites has been previously applied to infer some historical biogeographical routes of cyprinoid fish and their historical contacts, such as in the Balkans [12], in Iberian Peninsula [13] and in Northwest Africa [15], all these regions exhibiting a high diversity of endemic fish fauna. These studies revealed patterns of historical cyprinoid dispersion, continental associations and coevolutionary histories that included secondary contacts and host switching.

Benovics et al. [12] showed that, in the Balkans, the evolution of Dactylogyrus is associated with the historical dispersion and distribution of their cyprinoid hosts but is also affected by recent contacts between non-native and endemic cyprinoid species. Šimková 
et al. [15], using host-specific Dactylogyrus spp., supported the different origins of two Northwest African cyprinid lineages-Barbinae and Torinae-and inferred independent historical contacts between Iberian Luciobarbus (Barbinae) and two lineages of Northwest African cyprinids, these contacts were associated with host switches of Dactylogyrus parasites.

Benovics et al. [13] suggested multiple origins of the southern European Dactylogyrus spp. parasitizing cyprinids of Barbinae linked with the northern route of cyprinid dispersion [23] and the southern route via Northern Africa [24]. Their study highlighted the role of continental bridges between southern Europe and North Africa playing a crucial role in the historical dispersion of cyprinids and also affecting the distribution of host-specific parasites of Dactylogyrus. Finally, Benovics et al. [25] revealed that the Middle East represents the area of Dactylogyrus diversification and suggested that the attachment organ (termed the haptor) of each Dactylogyrus lineage has specific morphological characteristics that are associated with a particular dispersal event proposed for cyprinids.

In view of the demonstrated fact that host-specific Dactylogyrus parasites represent useful indicators of biogeographical contacts among cyprinoids, we focused on Dactylogyrus spp. parasitizing leuciscids of the Nearctic region and the European part of the Palearctic region. Therefore, the aim of our study was to investigate the phylogenetic position of Dactylogyrus spp. parasitizing North American leuciscids within Dactylogyrus phylogeny (in particular to investigate the phylogenetic relationships between Dactylogyrus spp. specific to North American leuciscids and those specific to European leuciscid hosts) and to resolve the origin of North American Dactylogyrus. We hypothesized that phylogenetic relationships among Dactylogyrus spp. parasitizing Holarctic leuciscids will reflect the historical biogeography of a fish fauna divided between two continents.

\section{Material and Methods}

\subsection{Parasite Collection}

Fish hosts were collected in 2018 and 2019 from four states in the United States: Arkansas, Mississippi, New York and Wisconsin (Table 1). Specimens of Dactylogyrus species used in this study were extracted from the gills of freshly killed euthanized cypriniform fish and were subsequently examined using fine needles and a dissecting microscope. Cypriniform species were determined by our local collaborators (included in acknowledgements) or with the help of identification keys. One half of the monogenean (either the posterior part with haptoral sclerites or anterior part containing the male copulatory organ) was placed in a $1.5 \mathrm{~mL}$ Eppendorf tube with 96\% ethanol for DNA extraction.

Table 1. List of cypriniform species collected from North America, including sites of collection, fish sample size and list of identified Dactylogyrus species.

\begin{tabular}{|c|c|c|c|c|c|}
\hline Fish Species & Country & Locality & Body Water & Sample Size & Dactylogyrus Species \\
\hline $\begin{array}{c}\text { Campostoma spadiceum } \\
\text { (Girard, 1856) }\end{array}$ & Arkansas & Polk County & Bear Creek & 11 & Dactylogyrus sp. 4 \\
\hline $\begin{array}{l}\text { Catostomus commersonii } \\
\text { (Lacepède, 1803) }\end{array}$ & New York & Cooperstown & Oaks Creek & 12 & Dactylogyrus sp. 8 \\
\hline Chrosomus neogaeus (Cope, 1867) & Wisconsin & Door County & Mink River & 13 & $\begin{array}{c}\text { D. cf. chrosomi, } \\
\text { Dactylogyrus sp. } 11\end{array}$ \\
\hline $\begin{array}{l}\text { Clinostomus elongatus } \\
\text { (Kirtland, 1840) }\end{array}$ & Wisconsin & Brown County & $\begin{array}{l}\text { Baird Creek, } \\
\text { Green Bay }\end{array}$ & 11 & D. confusus \\
\hline \multirow[t]{2}{*}{ Cyprinella venusta Girard, 1856} & Mississippi & $\begin{array}{l}\text { Oxbow south } \\
\text { of Cumbest } \\
\text { Bridge landing }\end{array}$ & Pascagoula River & 13 & D. venusti \\
\hline & Mississippi & Moon Lake & Pascagoula River & 12 & $\begin{array}{c}\text { Dactylogyrus sp. } 7 \\
\text { Dactylogyrus sp. } 2 \\
\text { variant B }\end{array}$ \\
\hline Cyprinella whipplei Girard, 1856 & Arkansas & Polk County & Caddo River & 2 & $\begin{array}{c}\text { Dactylogyrus sp. } 2 \\
\text { variant A }\end{array}$ \\
\hline
\end{tabular}


Table 1. Cont.

\begin{tabular}{|c|c|c|c|c|c|}
\hline Fish Species & Country & Locality & Body Water & Sample Size & Dactylogyrus Species \\
\hline $\begin{array}{l}\text { Hypentelium nigricans } \\
\text { (Lesueur, 1817) }\end{array}$ & Arkansas & $\begin{array}{l}\text { Montgomery } \\
\text { County }\end{array}$ & $\begin{array}{l}\text { Huddleston } \\
\text { Creek }\end{array}$ & 3 & $\begin{array}{l}\text { Dactylogyrus sp. 5, } \\
\text { Dactylogyrus sp. } 6\end{array}$ \\
\hline \multirow[t]{2}{*}{$\begin{array}{l}\text { Luxilus chrysocephalus } \\
\text { Rafinesque, } 1820\end{array}$} & Arkansas & Polk County & Caddo River & 3 & $\begin{array}{l}\text { D. arcus variant } \mathrm{A}, \mathrm{D} \text {. } \\
\text { semotilus variant } \mathrm{A} \text {, } \\
\text { Dactylogyrus sp. } 1 \text {, } \\
\text { Dactylogyrus sp. } 3\end{array}$ \\
\hline & Arkansas & Polk County & Big Fork Creek & 3 & D. perlus \\
\hline Luxilus cornutus (Mitchill, 1817) & Wisconsin & Brown County & West Twin River & 10 & $\begin{array}{l}\text { D. arcus variant } B, D \text {. } \\
\text { semotilus variant } B\end{array}$ \\
\hline $\begin{array}{l}\text { Nocomis biguttatus } \\
\text { (Kirtland, 1840) }\end{array}$ & Wisconsin & Brown County & West Twin River & 7 & $\begin{array}{c}\text { D. avinguis, } D \text {. } \\
\text { flagristylus, D. lachneri }\end{array}$ \\
\hline $\begin{array}{l}\text { Notemigonus crysoleucas } \\
\text { (Mitchill, 1814) }\end{array}$ & New York & Cooperstown & $\begin{array}{l}\text { Rom Hill Beaver } \\
\text { Pond }\end{array}$ & 5 & D. cf. parvicirrus \\
\hline Notropis petersoni Fowler, 1942 & Mississippi & & Bluff Creek & 2 & D. ornatus \\
\hline Opsopoeodus emiliae Hay, 1881 & Mississippi & & Bluff Creek & 1 & D. opsopoeodi \\
\hline \multirow[t]{2}{*}{$\begin{array}{l}\text { Pimephales notatus } \\
\text { (Rafinesque, 1820) }\end{array}$} & New York & Cooperstown & $\begin{array}{l}\text { Leatherstocking } \\
\text { Creek }\end{array}$ & 4 & $\begin{array}{l}\text { D. cf. atromaculatus } \\
\text { variant B }\end{array}$ \\
\hline & Arkansas & Polk County & Bear Creek & 2 & $\begin{array}{l}\text { D. bifurcatus, D. cf. } \\
\text { boopsi, D. cf. simplexus }\end{array}$ \\
\hline $\begin{array}{c}\text { Pimephales promelas Rafinesque, } \\
1820\end{array}$ & Wisconsin & Door County & $\begin{array}{l}\text { Hickory Oak } \\
\text { Pond }\end{array}$ & 7 & D. pectenatus \\
\hline $\begin{array}{l}\text { Rhinichthys atratulus } \\
\text { (Hermann, 1804) }\end{array}$ & Wisconsin & Brown County & $\begin{array}{l}\text { Baird Creek, } \\
\text { Green Bay }\end{array}$ & 2 & $\begin{array}{l}\text { D. cheloideus, D. } \\
\text { rhinichthius }\end{array}$ \\
\hline $\begin{array}{l}\text { Rhinichthys cataractae } \\
\text { (Valenciennes, 1842) }\end{array}$ & New York & Cooperstown & $\begin{array}{l}\text { Leatherstocking } \\
\text { Creek }\end{array}$ & 5 & $\begin{array}{l}\text { Dactylogyrus sp. } 9 \text {, } \\
\text { Dactylogyrus sp. } 10\end{array}$ \\
\hline \multirow[t]{2}{*}{$\begin{array}{l}\text { Semotilus atromaculatus } \\
\quad \text { (Mitchill, 1818) }\end{array}$} & Arkansas & Polk County & Big Fork Creek & 7 & $\begin{array}{l}\text { D. cf. atromaculatus } \\
\text { variant A }\end{array}$ \\
\hline & Wisconsin & Brown County & $\begin{array}{l}\text { Baird Creek, } \\
\text { Green Bay }\end{array}$ & 16 & $\begin{array}{l}\text { D. atromaculatus, } D . \\
\text { attenuatus }\end{array}$ \\
\hline
\end{tabular}

For each monogenean species, DNA sequencing of both specimens with the ethanolfixed posterior part and specimens with ethanol-fixed anterior part is important to avoid the misidentification of congeneric monogenean species exhibiting high similarities in attachment organ (posterior part) or in reproductive organs (anterior part). The other half of the worm was mounted on a slide and fixed with a mixture of glycerine and ammonium picrate (GAP) for species identification based on morphological characters (the sclerotized parts of the monogenean attachment organ (haptor), or the sclerotized parts of the reproductive organs-the copulatory organ and vaginal armaments). Dactylogyrus species were determined using available references [26-40].

\subsection{DNA Extraction, Amplification and Sequencing}

Ethanol preserved Dactylogyrus specimens were vacuum dried using a centrifugal vacuum concentrator (Eppendorf, Hamburg, Germany). Genomic DNA was extracted separately from each parasite specimen (1-10 specimens per species) using the DNeasy Blood \& Tissue Kit (Qiagen, Hilden, Germany) following the manufacturer's protocol. Two fragments of nuclear ribosomal DNA, generally considered suitable markers for monogenean species determination and widely applied in phylogenetic studies of Dactylogyrus [10,12-15,18,25], were analyzed as follows: (i) a fragment spanning partial $18 \mathrm{~S}$ rDNA and internal transcribed spacer (18S rDNA + ITS1); and (ii) a fragment of partial $28 \mathrm{~S}$ rDNA.

The partial 18S rDNA + ITS1 fragment was amplified using the primers S1 (forward, 5'-ATTCCGATAACGAACGAGACT-3') [41] and one of the newly designed reverse primers DactR1 (reverse, $5^{\prime}$-GAGCCGAGTGATCCACCACT-3') or DactR2 (reverse, $5^{\prime}$-GTTCACACAGTTTGCTGCACT-3'). The second fragment, partial $28 \mathrm{~S}$ rDNA, was amplified using primers $\mathrm{C} 1$ (forward, $5^{\prime}$-ACCCGCTGAATTTAAGCA-3') and D2 (reverse, 
5'-TGGTCCGTGTTTCAAGAC-3') [42]. Each amplification reaction contained $1 \mathrm{U}$ of Taq polymerase (Fermentas), $1 \times$ PCR buffer (Fermentas), $1.5 \mathrm{mM} \mathrm{MgCl}_{2}, 200 \mu \mathrm{M}$ of each dNTP, $0.5 \mu \mathrm{M}$ (for $28 \mathrm{~S} \mathrm{rDNA}$ ) or $0.8 \mu \mathrm{M}$ (for the fragment including $18 \mathrm{~S}$ rDNA and ITS1) of each primer and $5 \mu \mathrm{L}$ of DNA extract (corresponding to $20 \mathrm{ng} / \mu \mathrm{L}$ ).

For DNA amplification, the following PCR conditions were used: initial denaturation at $94{ }^{\circ} \mathrm{C}$ for $2 \mathrm{~min}, 39$ cycles of denaturation at $94{ }^{\circ} \mathrm{C}$ for $60 \mathrm{~s}$ (for $18 \mathrm{~S} \mathrm{rDNA}+\mathrm{ITS} 1$ ) or $20 \mathrm{~s}$ (for $28 \mathrm{~S}$ rDNA), annealing at $53{ }^{\circ} \mathrm{C}$ for $60 \mathrm{~s}$ (for $18 \mathrm{~S} \mathrm{rDNA}+\mathrm{ITS} 1$ ) or at $56{ }^{\circ} \mathrm{C}$ for $30 \mathrm{~s}$ (for $28 \mathrm{~S}$ rDNA), an extension at $72{ }^{\circ} \mathrm{C}$ for $90 \mathrm{~s}$ and a final extension at $72{ }^{\circ} \mathrm{C}$ for $10 \mathrm{~min}$. The PCR products were checked by electrophoresis in $2 \%$ agarose gel and purified using ExoSap (Ecoli, Bratislava, Slovakia).

Sequencing was performed on an ABI 3130 DNA Genetic Analyzer (Applied Biosystems) using the BigDye Terminator v3.1 Cycle Sequencing Kit (Applied Biosystems, Foster City, CA, USA) and the same primers as those for PCR. Forward and reverse sequences were visually inspected, edited and combined into contigs using the software Sequencher (Ann Arbor, MI, USA). Newly generated sequences were deposited in GenBank (see Table 2 for accession numbers).

\subsection{Phylogenetic Reconstruction}

Phylogenetic analyses were performed using two sequence datasets. The first dataset included 31 partial 28S rDNA sequences of North American Dactylogyrus species obtained in the present study (only one genetic variant was included for species with intraspecific variability, i.e., D. arcus, D. semotilus and Dactylogyrus sp. 2, and no sequence for $D$. cf. atromaculatus was included in this dataset) and 37 sequences of Dactylogyrus species retrieved from GenBank representing four phylogenetic Dactylogyrus lineages revealed by Šimková et al. [15] (see Table 2 for accession numbers). Two species of Dactylogyridae, Aliatrema cribbi (acc. no. AY820612) and Euryhaliotrematoides pirulum (acc. no. AY820618), were selected as the outgroup.

The second dataset was based on concatenated data of partial 18S rDNA + ITS1 and $28 \mathrm{~S}$ rDNA and included 36 sequences of North American Dactylogyrus species (all genetic variants for North American Dactylogyrus species exhibiting intraspecific variability were included) and the sequences of 17 selected Dactylogyrus species parasitizing European fishes of Leuciscidae and Cyprinidae [15]. Mid-point rooting was applied because of the ambiguous positions of lineages II and lineages III in relation to lineage IV revealed by previous phylogenetic studies $[15,18,25]$. Sequence divergence for the North American species exhibiting intraspecific variability between different localities was estimated in MEGA X [43] using the p-distance model.

Phylogenetic analyses were conducted using the Maximum Likelihood (ML) and Bayesian Inference (BI) methods. Sequence alignments were performed separately for each gene in MAFFT v. 7 https://mafft.cbrc.jp/ (accessed on 15 May 2021) [44] with the G-INS-i algorithm. Gaps, hypervariable regions and ambiguously aligned regions were removed from the alignments using GBlocks v. 0.91b http:/ / phylogeny.lirmm.fr / phylo_cgi/one_task.cgi?task_type=gblocks (accessed on 20 May 2021) [45]. Model selection was performed for each alignment partition, and the following models were selected using the Bayesian information criterion (BIC) in jModelTest v. 2.1.10 https: / github.com/ ddarriba/jmodeltest2 (accessed on 20 May 2021) [46]: the 28S rDNA dataset: GTR + I + G; the concatenated dataset: TPM3 + I + G for $18 \mathrm{~S}$ rDNA, SYM + I + G for ITS1 and GTR + I + $\mathrm{G}$ for $28 \mathrm{~S}$ rDNA. 
Table 2. List of Dactylogyrus species used in phylogenetic analyses, their fish host species, country of collection and GenBank accession numbers for DNA sequences.

\begin{tabular}{|c|c|c|c|c|c|c|}
\hline \multirow{2}{*}{ Dactylogyrus Species } & \multirow{2}{*}{ Cypriniform Host Species } & \multirow{2}{*}{$\begin{array}{l}\text { Cypriniform } \\
\text { Family }\end{array}$} & \multirow{2}{*}{$\begin{array}{l}\text { Cypriniform } \\
\text { Subfamily }\end{array}$} & \multirow{2}{*}{$\begin{array}{c}\text { Sampling } \\
\text { Locality }\end{array}$} & \multicolumn{2}{|c|}{ GenBank Accession No. } \\
\hline & & & & & $28 \mathrm{~S}$ rDNA & 18S rDNA and ITS1 \\
\hline \multicolumn{7}{|l|}{ Lineage I } \\
\hline D. doadrioi El Gharbi, Renaud \& Lambert, 1993 & Luciobarbus guiraonis (Steindachner, 1866) & Cyprinidae & Barbinae & Spain & KY629346 & - \\
\hline D. labei Musselius \& Gusev, in Gusev, 1976 & Gibelion catla (Hamilton, 1822) & Cyprinidae & Labeoninae & India & JX566720 & - \\
\hline D. mascomai El Gharbi, Renaud \& Lambert, 1992 & Luciobarbus guiraonis (Steindachner, 1866) & Cyprinidae & Barbinae & Spain & KY629348 & - \\
\hline D. quanfami Ha Ky, 1971 & Cirrhinus molitorella (Valenciennes, 1844) & Cyprinidae & Labeoninae & China & EF100536 & - \\
\hline D. volutus El Gharbi, Birgi \& Lambert, 1994 & Carasobarbus fritschii (Günther, 1874) & Cyprinidae & Torinae & Morocco & KY629353 & - \\
\hline D. zatensis El Gharbi, Birgi \& Lambert, 1994 & Carasobarbus fritschii & Cyprinidae & Torinae & Morocco & KY629352 & - \\
\hline \multicolumn{7}{|l|}{ Lineage II } \\
\hline D. bicornis Malewitzkaja, 1941 & Rhodeus meridionalis Karaman, 1924 & Acheilognathidae & - & Greece & KY629345 & - \\
\hline D. cryptomeres Bychowsky, 1934 & Gobio gobio (Linnaeus, 1758) & Gobionidae & - & $\begin{array}{l}\text { Czech } \\
\text { Republic }\end{array}$ & AJ969947 & - \\
\hline D. hypophthalmichthys Akhmerov, 1952 & $\begin{array}{l}\text { Hypophthalmichthys molitrix } \\
\text { (Valenciennes, 1844) }\end{array}$ & Xenocyprididae & - & China & EF100532 & - \\
\hline D. lamellatus Akhmerow, 1952 & Ctenopharyngodon idella (Valenciennes, 1844) & Xenocyprididae & - & China & AY307019 & - \\
\hline D. parabramis Akhmerov, 1952 & Megalobrama terminalis (Richardson, 1846) & Xenocyprididae & - & China & EF100534 & - \\
\hline D. petruschewskyi Gusev, 1955 & Megalobrama amblycephala Yih, 1955 & Xenocyprididae & - & China & AY548927 & - \\
\hline \multicolumn{7}{|l|}{ Lineage III } \\
\hline D. anchoratus (Dujardin, 1845) Wagener, 1857 & Carassius gibelio (Bloch, 1782) & Cyprinidae & Cyprininae & Croatia & KY863555 & - \\
\hline D. aspili Birgi \& Lambert, 1987 & Enteromius macrops (Boulenger, 1911) & Cyprinidae & Smiliogastrinae & Senegal & KY629359 & - \\
\hline D. extensus Mueller \& Van Cleave, 1932 & Cyprinus carpio Linnaeus, 1758 & Cyprinidae & Cyprininae & $\begin{array}{l}\text { Czech } \\
\text { Republic }\end{array}$ & AJ969944 & - \\
\hline D. marocanus El Gharbi, Birgi \& Lambert, 1994 & Labeobarbus maroccanus (Günther, 1902) & Cyprininae & Torinae & Morocco & MW218579 & - \\
\hline D. oligospirophallus Paperna, 1973 & Labeo coubie Rüppell, 1832 & Cyprinidae & Labeoninae & Senegal & KY629361 & - \\
\hline D. senegalensis Paperna, 1969 & Labeo senegalensis Valenciennes, 1842 & Cyprinidae & Labeoninae & Senegal & KY629363 & - \\
\hline D. titus Guegan, Lambert \& Euzet, 1988 & Labeo senegalensis & Cyprinidae & Labeoninae & Senegal & KY629364 & - \\
\hline D. vastator Nybelin, 1924 & Carassius gibelio (Bloch, 1782) & Cyprinidae & Cyprininae & $\begin{array}{l}\text { Czech } \\
\text { Republic }\end{array}$ & KY629366 & - \\
\hline \multicolumn{7}{|l|}{ Lineage IV } \\
\hline $\begin{array}{l}\text { Dactylogyrus andalousiensis El Gharbi, Renaud \& } \\
\text { Lambert, } 1993\end{array}$ & Luciobarbus sclateri (Günther, 1868) & Cyprinidae & Barbinae & Portugal & KY629351 & KY629331 \\
\hline Dactylogyrus borealis Nybelin, 1937 & Phoxinus bigerri Kottelat, 2007 & Leuciscidae & Phoxininae & Spain & MN338222 & MN365688 \\
\hline
\end{tabular}


Table 2. Cont.

\begin{tabular}{|c|c|c|c|c|c|c|}
\hline \multirow{2}{*}{ Dactylogyrus Species } & \multirow{2}{*}{ Cypriniform Host Species } & \multirow{2}{*}{$\begin{array}{c}\text { Cypriniform } \\
\text { Family }\end{array}$} & \multirow{2}{*}{$\begin{array}{l}\text { Cypriniform } \\
\text { Subfamily }\end{array}$} & \multirow{2}{*}{$\begin{array}{l}\text { Sampling } \\
\text { Locality }\end{array}$} & \multicolumn{2}{|c|}{ GenBank Accession No. } \\
\hline & & & & & 28S rDNA & 18S rDNA and ITS1 \\
\hline Dactylogyrus carpathicus Zakhvatkin, 1951 & Barbus barbus (Linnaeus, 1758) & Cyprinidae & Barbinae & $\begin{array}{l}\text { Czech } \\
\text { Republic }\end{array}$ & KY201111 & KY201098 \\
\hline Dactylogyrus cornu Linstow, 1878 & Vimba vimba (Linnaeus, 1758) & Leuciscidae & Leuciscinae & $\begin{array}{l}\text { Czech } \\
\text { Republic }\end{array}$ & KY629371 & KY629342 \\
\hline Dactylogyrus crucifer Wagener, 1857 & Rutilus rutilus (Linnaeus, 1758) & Leuciscidae & Leuciscinae & $\begin{array}{l}\text { Czech } \\
\text { Republic }\end{array}$ & KY629374 & AJ564120 \\
\hline Dactylogyrus ergensi Molnár, 1964 & Chondrostoma nasus (Linnaeus, 1758) & Leuciscidae & Leuciscinae & Greece & MG792989 & MG792874 \\
\hline $\begin{array}{c}\text { Dactylogyrus legionensis Gonzalez Lanza \& } \\
\text { Alvarez Pellitero, } 1982\end{array}$ & Luciobarbus guiraonis (Steindachner, 1866) & Cyprinidae & Barbinae & Spain & KY629350 & KY629330 \\
\hline Dactylogyrus malleus Linstow, 1877 & Barbus barbus & Cyprinidae & Barbinae & $\begin{array}{l}\text { Czech } \\
\text { Republic }\end{array}$ & KY201112 & KY201099 \\
\hline Dactylogyrus martinovici Ergens, 1970 & Pachychilon pictum (Heckel \& Kner, 1858) & Leuciscidae & Leuciscinae & Albania & MG793000 & MG792884 \\
\hline Dactylogyrus nanus Dogiel \& Bychowsky, 1934 & Rutilus rutilus & Leuciscidae & Leuciscinae & $\begin{array}{l}\text { Czech } \\
\text { Republic }\end{array}$ & AJ969942 & AJ564145 \\
\hline Dactylogyrus petkovici Ergens, 1970 & Pachychilon pictum & Leuciscidae & Leuciscinae & Albania & MG793002 & MG792886 \\
\hline $\begin{array}{c}\text { Dactylogyrus scorpius Rahmouni, Řehulková \& } \\
\text { Simková, } 2017\end{array}$ & $\begin{array}{c}\text { Luciobarbus rifensis Doadrio, Casal-Lopez \& } \\
\text { Yahyaoui, } 2015\end{array}$ & Cyprinidae & Barbinae & Morocco & KX553860 & KX578023 \\
\hline Dactylogyrus sphyrna Linstow, 1878 & Rutilus rutilus & Leuciscidae & Leuciscinae & $\begin{array}{l}\text { Czech } \\
\text { Republic }\end{array}$ & AJ969943 & AJ564154 \\
\hline $\begin{array}{c}\text { Dactylogyrus varius Rahmouni, Řehulková \& } \\
\text { Šimková, } 2017\end{array}$ & $\begin{array}{c}\text { Luciobarbus maghrebensis Doadrio, Perea \& } \\
\text { Yahyaoui, } 2015\end{array}$ & Cyprinidae & Barbinae & Morocco & KX553863 & KX578026 \\
\hline Dactylogyrus vistulae Prost, 1957 & Squalius prespensis (Fowler, 1977) & Leuciscidae & Leuciscinae & Albania & KY629369 & KY629340 \\
\hline D. arcus Rogers, 1967 (variant A) & Luxilus chrysocephalus Rafinesque, 1820 & Leuciscidae & Pogonichthyinae & Arkansas & OM108517 & OM108553 \\
\hline D. arcus Rogers, 1967 (variant B) & Luxilus sp. & Leuciscidae & Pogonichthyinae & Wisconsin & OM108518 & OM108554 \\
\hline D. atromaculatus Mizelle, 1938 & Semotilus atromaculatus & Leuciscidae & Plagopterinae & Wisconsin & OM108519 & OM108555 \\
\hline D. cf. atromaculatus Mizelle, 1938 (variant A) & Semotilus atromaculatus (Mitchill, 1818) & Leuciscidae & Plagopterinae & Arkansas & OM108523 & OM108559 \\
\hline D. cf. atromaculatus Mizelle, 1938 (variant B) & Pimephales notatus (Rafinesque, 1820) & Leuciscidae & Pogonichthyinae & New York & OM108524 & OM108560 \\
\hline D. attenuatus Mizelle \& Klucka, 1953 & Semotilus atromaculatus & Leuciscidae & Plagopterinae & Wisconsin & OM108520 & OM108556 \\
\hline D. aviunguis Chien, 1974 & Nocomis biguttatus (Kirtland, 1840) & Leuciscidae & Pogonichthyinae & Wisconsin & OM108521 & OM108557 \\
\hline D. bifurcatus Mizelle, 1937 & Pimephales notatus & Leuciscidae & Pogonichthyinae & Arkansas & OM108522 & OM108558 \\
\hline D. cf. boopsi Cloutman, 1994 & Pimephales notatus & Leuciscidae & Pogonichthyinae & Arkansas & OM108525 & OM108561 \\
\hline D. cheloideus Rogers, 1967 & Rhinichthys atratulus (Hermann, 1804) & Leuciscidae & Pogonichthyinae & Wisconsin & OM108531 & OM108567 \\
\hline
\end{tabular}


Table 2. Cont.

\begin{tabular}{|c|c|c|c|c|c|c|}
\hline \multirow{2}{*}{ Dactylogyrus Species } & \multirow{2}{*}{ Cypriniform Host Species } & \multirow{2}{*}{$\begin{array}{l}\text { Cypriniform } \\
\text { Family }\end{array}$} & \multirow{2}{*}{$\begin{array}{l}\text { Cypriniform } \\
\text { Subfamily }\end{array}$} & \multirow{2}{*}{$\begin{array}{l}\text { Sampling } \\
\text { Locality }\end{array}$} & \multicolumn{2}{|c|}{ GenBank Accession No. } \\
\hline & & & & & $28 \mathrm{~S}$ rDNA & 18S rDNA and ITS1 \\
\hline D. cf. chrosomi Hanek, Molnár \& Fernando, 1975 & Chrosomus neogaeus (Cope, 1867) & Leuciscidae & Laviniinae & Wisconsin & OM108526 & OM108562 \\
\hline D. confusus Mueller, 1938 & Clinostomus elongatus (Kirtland, 1840) & Leuciscidae & Pogonichthyinae & Wisconsin & OM108529 & OM108565 \\
\hline D. lachneri Chien, 1971 & Nocomis biguttatus & Leuciscidae & Pogonichthyinae & Wisconsin & OM108532 & OM108568 \\
\hline D. opsopoeodi Rogers, 1967 & Opsopoeodus emiliae Hay, 1881 & Leuciscidae & Pogonichthyinae & Mississippi & OM108533 & OM108569 \\
\hline D. ornatus Rogers, 1967 & Notropis petersoni Fowler, 1942 & Leuciscidae & Pogonichthyinae & Mississippi & OM108534 & OM108570 \\
\hline D. cf. parvicirrus Seamster, 1948 & Notemigonus crysoleucas (Mitchill, 1814) & Leuciscidae & Leuciscinae & New York & OM108527 & OM108563 \\
\hline D. perlus Mueller, 1938 & Luxilus chrysocephalus & Leuciscidae & Pogonichthyinae & Arkansas & OM108536 & OM108572 \\
\hline D. rhinichthius Wood \& Mizelle, 1957 & Rhinichthys atratulus & Leuciscidae & Pogonichthyinae & Wisconsin & OM108537 & OM108573 \\
\hline D. semotilus Wood \& Mizelle, 1957 (variant A) & Luxilus chrysocephalus & Leuciscidae & Pogonichthyinae & Arkansas & OM108538 & OM108574 \\
\hline D. semotilus Wood \& Mizelle, 1957 (variant B) & Luxilus sp. & Leuciscidae & Pogonichthyinae & Wisconsin & OM108539 & OM108575 \\
\hline D. cf. simplexus Monaco \& Mizelle, 1955 & Pimephales notatus & Leuciscidae & Pogonichthyinae & Arkansas & OM108528 & OM108564 \\
\hline D. venusti Rogers, 1967 & Cyprinella venusta Girard, 1856 & Leuciscidae & Pogonichthyinae & Mississippi & OM108552 & OM108588 \\
\hline Dactylogyrus sp. 1 & Luxilus chrysocephalus & Leuciscidae & Pogonichthyinae & Arkansas & OM108540 & OM108576 \\
\hline Dactylogyrus sp. 2 variant A & Cyprinella whipplei Girard, 1856 & Leuciscidae & Pogonichthyinae & Arkansas & OM108541 & OM108577 \\
\hline Dactylogyrus sp. 2 variant B & Cyprinella venusta & Leuciscidae & Pogonichthyinae & Mississippi & OM108542 & OM108578 \\
\hline Dactylogyrus sp. 4 & Campostoma spadiceum (Girard, 1856) & Leuciscidae & Pogonichthyinae & Arkansas & OM108544 & OM108580 \\
\hline Dactylogyrus sp. 5 & Hypentelium nigricans (Lesueur, 1817) & Catostomidae & Catostominae & Arkansas & OM108545 & OM108581 \\
\hline Dactylogyrus sp. 6 & Hypentelium nigricans & Catostomidae & Catostominae & Arkansas & OM108546 & OM108582 \\
\hline Dactylogyrus sp. 7 & Cyprinella venusta & Leuciscidae & Pogonichthyinae & Mississippi & OM108547 & OM108583 \\
\hline Dactylogyrus sp. 8 & Catostomus commersonii (Lacepède, 1803) & Catostomidae & Catostominae & New York & OM108548 & OM108584 \\
\hline Dactylogyrus sp. 9 & Rhinichthys cataractae (Valenciennes, 1842) & Leuciscidae & Pogonichthyinae & New York & OM108549 & OM108585 \\
\hline Dactylogyrus sp. 10 & Rhinichthys cataractae & Leuciscidae & Pogonichthyinae & New York & OM108550 & OM108586 \\
\hline Dactylogyrus sp. 11 & Chrosomus neogaeus & Leuciscidae & Laviniinae & Wisconsin & OM108551 & OM108587 \\
\hline
\end{tabular}


ML phylogenetic reconstruction was performed using the IQ-TREE v. 1.6.12 [47] on the W-IQ-TREE webserver (http:/ / iqtree.cibiv.univie.ac.at (accessed on 20 May 2021) [48]. Branch support was estimated using ultrafast bootstrap approximation [49] with 10,000 replicates. BI analyses were conducted using MrBayes v. 3.2.1 https:/ / nbisweden.github. io/MrBayes (accessed on 30 May 2021) [50]. Four simultaneous chains (one cold and three heated) of the Markov Chain Monte Carlo (MCMC) algorithm were run twice for $10^{7}$ generations. Tree topologies were sampled every 100 generations, whereby the first $30 \%$ of trees from each run were discarded as "burn-in" to obtain the consensus tree and posterior probability values (PP). The convergence (where the average standard deviation of the split frequencies was lower than 0.01) and effective sampling sizes of all parameters were checked in Tracer v. 1.7.1 https: / / github.com/beast-dev/tracer/releases/latest (accessed on 5 June 2021) [51]. Trees were visualized and edited using FigTree v. 1.4.4 http:/ / tree.bio. ed.ac.uk/software/figtree (accessed on 15 June 2021) [52].

Character states were mapped onto the phylogenetic trees as follows: First, states representing the character reflecting the geographical distribution of cypriniform species, and states representing different cyprinoid lineages (cyprinoid families) and the catostomid lineage were mapped onto the Dactylogyrus phylogeny that included all Dactylogyrus species analyzed.

Next, character states representing the clades of Leuciscidae (subfamilies within Leuciscidae, following Schönhuth et al. [21]) and cypriniform lineages-Catostomidae and Cyprinidae were mapped onto the phylogenetic reconstruction that included the data set of Dactylogyrus species of Leuciscidae, three Dactylogyrus species of Catostomidae and a few Dactylogyrus species of Cyprinidae previously shown to be nested within the Dactylogyrus of European Leuciscidae [15]. The mapping was performed in Mesquite v. 3.2 https: / / www.mesquiteproject.org/ (accessed on 15 Jun 2021) [53].

\section{Results}

\subsection{Dactylogyrus Species of Nearctic Cypriniform Fish}

A total of 32 Dactylogyrus species from a total of 18 cypriniform host species (16 species of Leuciscidae and two species of Catostomidae) were recognized on the basis of morphological features (Table 2). For four Dactylogyrus species, multiple genetic variants were found. More specifically, two genetic variants were found for $D$. arcus (with the following p-distances: $0.4 \%$ for $18 \mathrm{~S}$ rDNA, $1 \%$ for ITS1 and $0.1 \%$ for $28 \mathrm{~S}$ rDNA) and D. semotilus (with $p$-distance $=0.1 \%$ for $28 \mathrm{~S}$ rDNA), each of them parasitizing two Luxilus species-one from a northern locality (Wisconsin) and another from a southern locality (Arkansas).

Two genetic variants were also found for Dactylogyrus sp. 2 parasitizing Cyprinella whipplei and C. venusta from two southern localities (Arkansas and Mississippi) (with the following p-distances: $0.1 \%$ for $28 \mathrm{~S}$ rDNA and $0.5 \%$ for ITS1), and two genetic variants were found for D. cf. atromaculatus parasitizing Semotilus atromaculatus from a southern locality (Arkansas) and Pimephales notatus collected in a northern locality (New York) (with $p$-distance $=2.1 \%$ for ITS1).

The richness of Dactylogyrus species on the fish investigated was very low; eight fish species were parasitized by a single Dactylogyrus species, and five fish species were parasitized by two Dactylogyrus species. Five fish species were parasitized by more than two Dactylogyrus species, i.e., from three to five Dactylogyrus species per fish species with Luxilus chrysocephalus and P. notatus exhibiting higher Dactylogyrus diversity in our sample (Table 1). Different Dactylogyrus species were found on the same host species collected from different localities (C. venusta, L. chrysocephalus, P. notatus and S. atromaculatus). Dactylogyrus species also exhibited high host specificity, i.e., except for four Dactylogyrus species with intraspecific variability, each Dactylogyrus species was strictly host specific (i.e., parasitizing a single host species). 


\subsection{Phylogenetic Position of Neartic Dactylogyrus Species within the Dactylogyrus Phylogeny}

ML and BI analyses based on aligned partial 28S rDNA sequences yielded phylogenetic trees with mostly similar branching topologies and congruent nodal support values (Figure 1). Essentially, four Dactylogyrus lineages were recognized (lineages I-IV). The first was a well-supported lineage included species parasitizing Asian Cyprinidae (representative of Labeoninae) and a monophyletic group of Dactylogyrus spp. parasitizing fishes of the African Torinae and Iberian Barbinae (both Cyprinidae). However, the position of D. labei on Asian Labeoninae within lineage I was not supported.

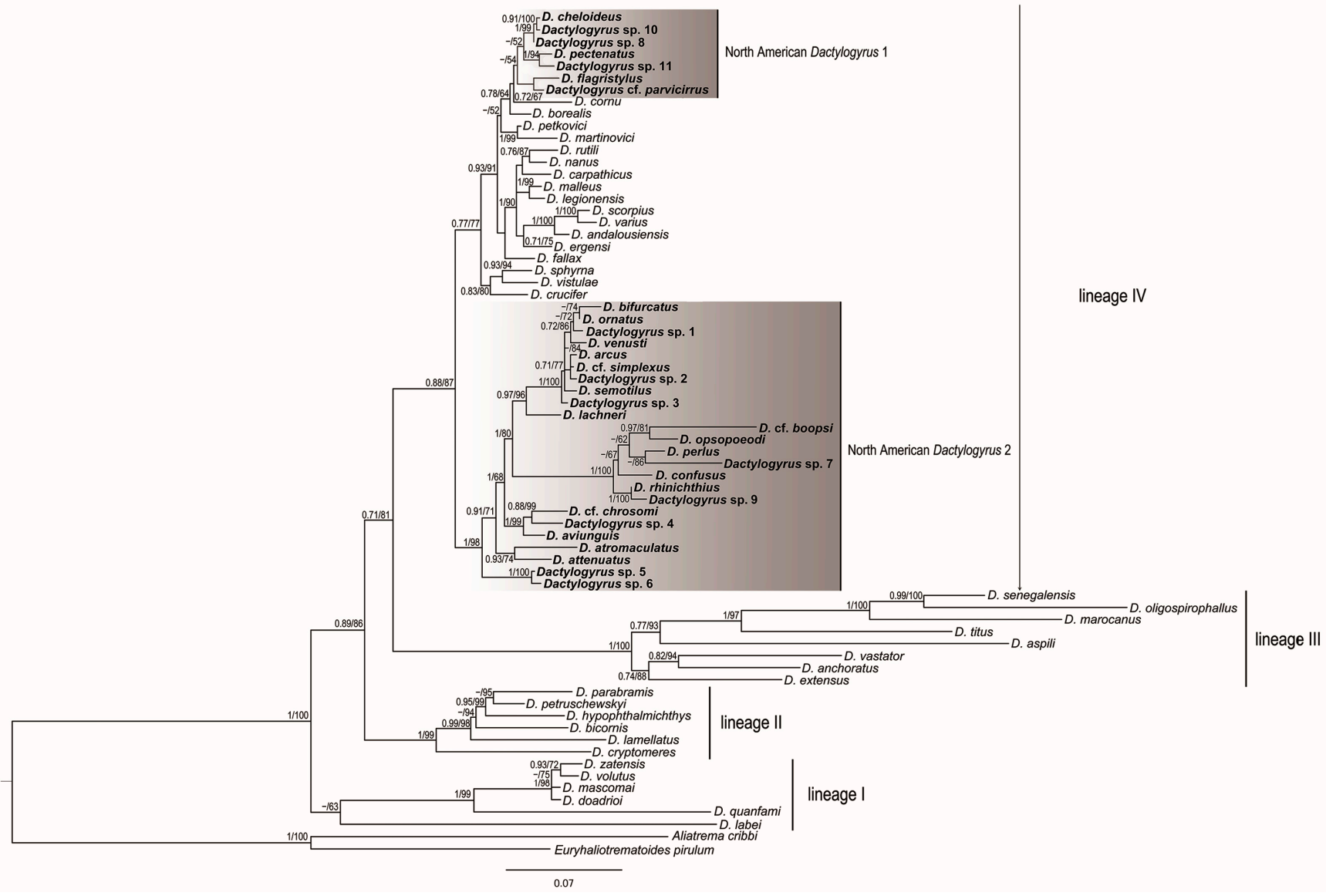

Figure 1. BI tree inferred from the analyses of partial 28S rDNA sequences of Dactylogyrus species. Numbers along the branches indicate posterior probabilities and bootstrap values resulting from BI and ML analyses. Only values $>0.70$ for $\mathrm{BI}$ and $>50 \%$ for $\mathrm{ML}$ are shown. New sequences generated in the present study are shown in bold.

Lineage I was sister to a clade of Dactylogyrus spp. including lineages II, III and IV. Lineage II represented Dactylogyrus species parasitizing fish species belonging to Gobionidae, Xenocyprinidae and Acheilognathidae. Lineage III was formed by Dactylogyrus species parasitizing fish species of Cyprinidae-more specifically, Cyprininae with a Euro-Asian distribution and likely of Asian origin and African Labeoninae. Lineage IV was a large lineage that included Dactylogyrus species distributed on Nearctic Leuciscidae as well as three Dactylogyrus species parasitizing two species of Catostomidae (North American Dactylogyrus species were included in two clades, see Figure 1), Dactylogyrus species restricted to Palearctic Leuciscidae (in our phylogenetic analyses, restricted to European samples) and some Dactylogyrus species on Cyprinidae (European and North African Barbinae).

ML and BI analyses based on the alignment of concatenated data including partial $28 \mathrm{~S}$ rDNA, partial 18S rDNA and ITS1 of Dactylogyrus species belonging to lineage IV yielded phylogenetic trees with similar branching topologies and congruent support values 
(Figure 2). Two clades were clearly recognized. One included strictly Nearctic Dactylogyrus and was formed by four well-supported subgroups, whereas the other included Nearctic Dactylogyrus and Palearctic Dactylogyrus parasitizing European Leuciscidae and Cyprinidae (Barbinae).

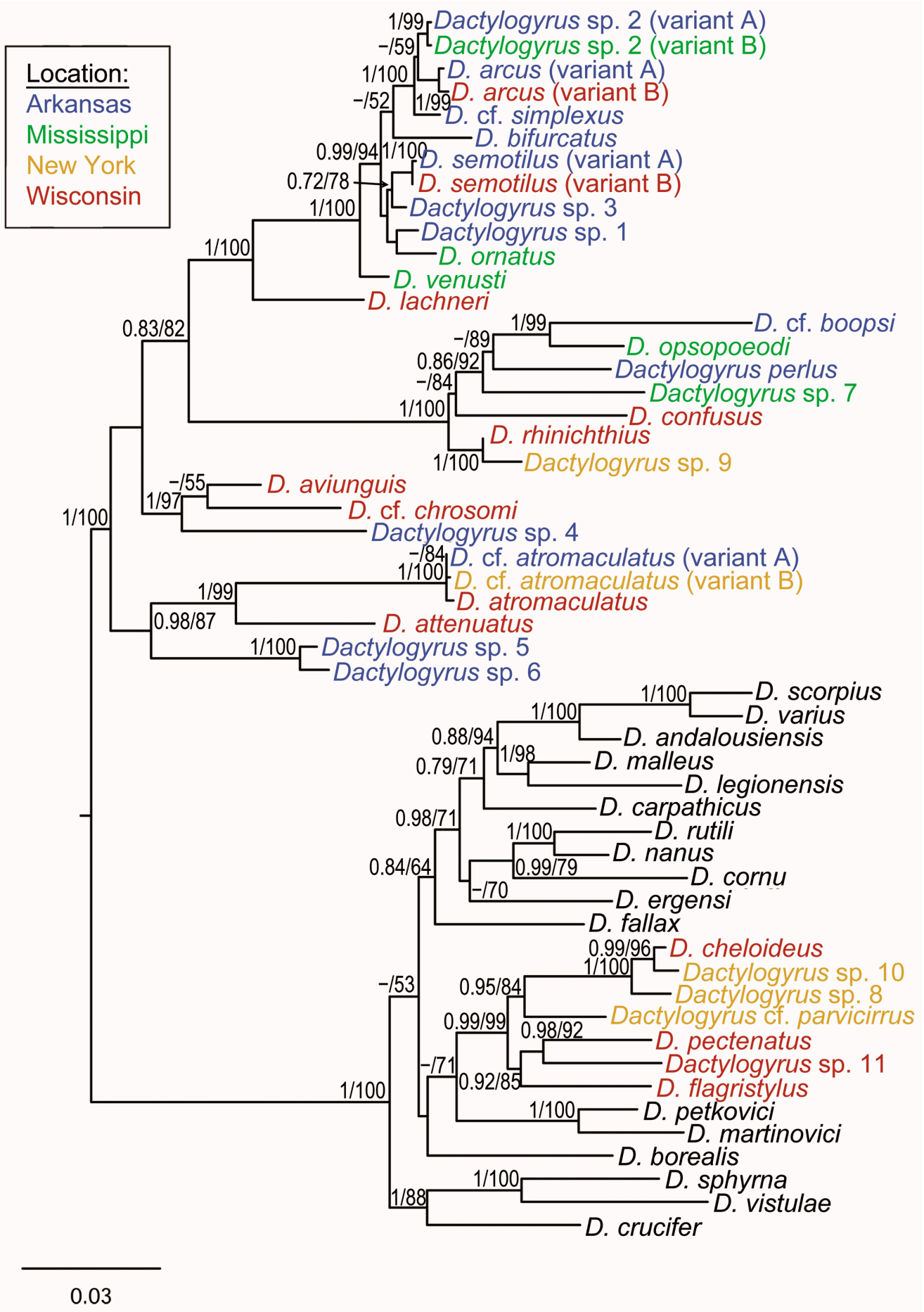

Figure 2. BI tree inferred from analyses of concatenated 18S rRNA, ITS1 and 28S rDNA sequences of Dactylogyrus species of lineage IV. Numbers along the branches indicate posterior probabilities and bootstrap values resulting from BI and ML analyses. Only values $>0.70$ for $\mathrm{BI}$ and $>50 \%$ for ML are shown. Localities of collections for North American Dactylogyrus species are highlighted by different colors. 
In this clade, a monophyletic group of exclusively Nearctic Dactylogyrus was nested within Palearctic Dactylogyrus; however, the phylogenetic relationships between this Nearctic Dactylogyrus group and two European species D. petkovici and D. martinovici parasitizing Balkan endemic leuciscids was only weakly supported by ML analysis, and the phylogenetic position of $D$. borealis parasitizing Phoxinus phoxinus was not supported.

\subsection{Origin of Nearctic Dactylogyrus}

The mapping of the geographical distribution of fish hosts onto the phylogeny of all four Dactylogyrus lineages (Figure 3) clearly indicated two independent origins of Nearctic Dactylogyrus. North American Dactylogyrus 1 (as defined in Figure 1) originated from Europe, whilst the origin of North American Dactylogyrus 2 was not fully resolved on the basis of the area mapped. The mapping of fish families (cyprinoid families and Catostomidae) onto the phylogeny of all four Dactylogyrus lineages (Figure 4) showed that Leuciscidae were colonized by Dactylogyrus from Cyprinidae, and Catostomidae were colonized twice from Leuciscidae.

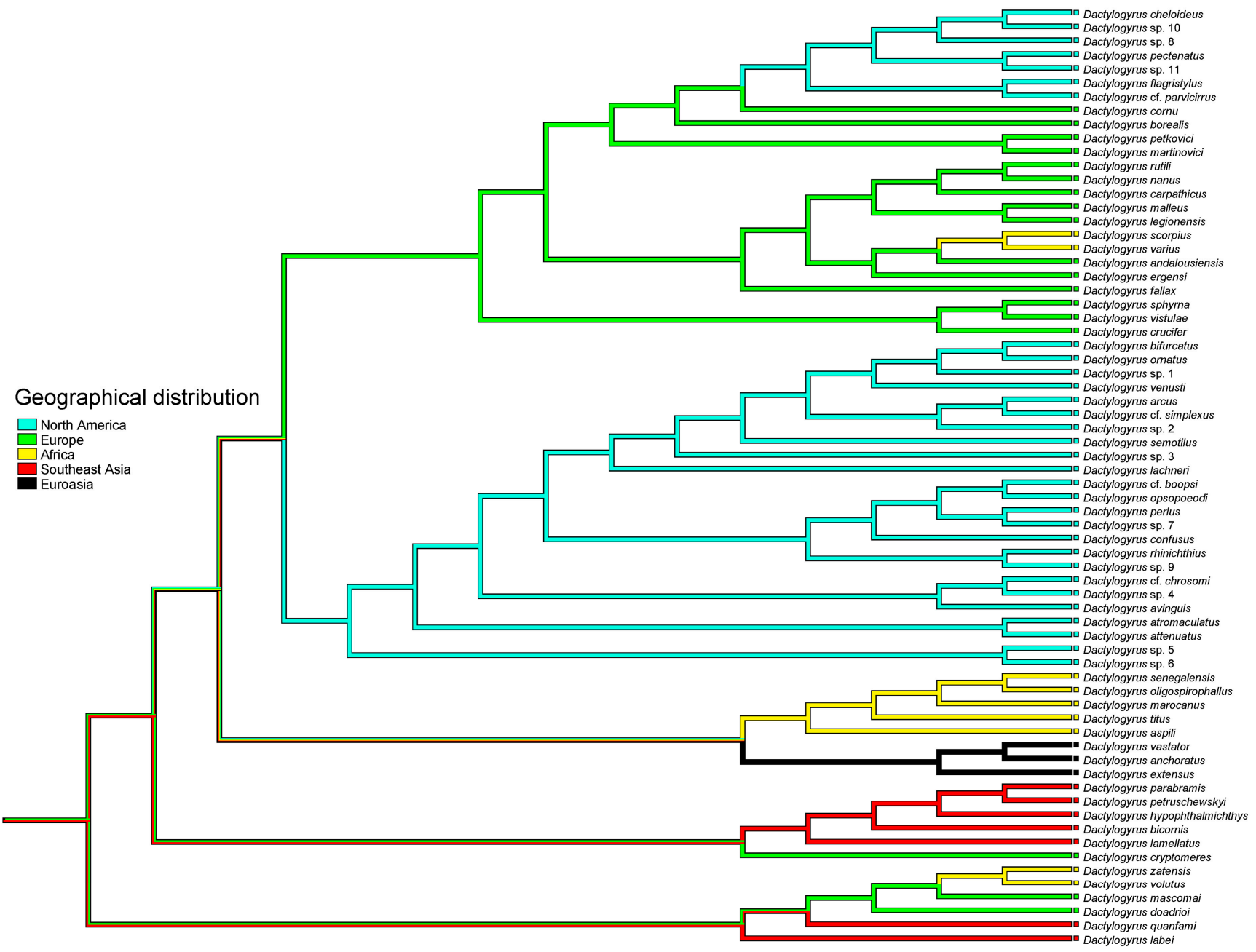

Figure 3. Mapping of the geographical distribution of fish hosts onto the BI reconstruction of Dactylogyrus phylogeny.

The mapping of leuciscid clades (i.e., at the level of subfamilies) defined by Schönhuth et al. (2018) onto lineage IV of Dactylogyrus (Figure 5) clearly indicated that the OW clade (Leuciscinae) is an ancestral host group for Dactylogyrus of the group, including North American clade 1. Within North American Dactylogyrus 1, colonization from the OW clade (Leuciscinae) to the NA clade (Pogonichthyinae) was evidenced. Moreover, our mapping revealed two independent colonisations of the WC clade (Laviniinae represented 
by Chrosomus neogaeus in our data) by Dactylogyrus parasitizing fish of the NA clade. The origin of Dactylogyrus cf. parvicirrus parasitizing a single Nearctic representative of the OW clade (Notemigonus crysoleucas) was unclear-either this species was colonized by Dactylogyrus from European Leuciscinae or from Nearctic Pogonichthyinae (NA clade).

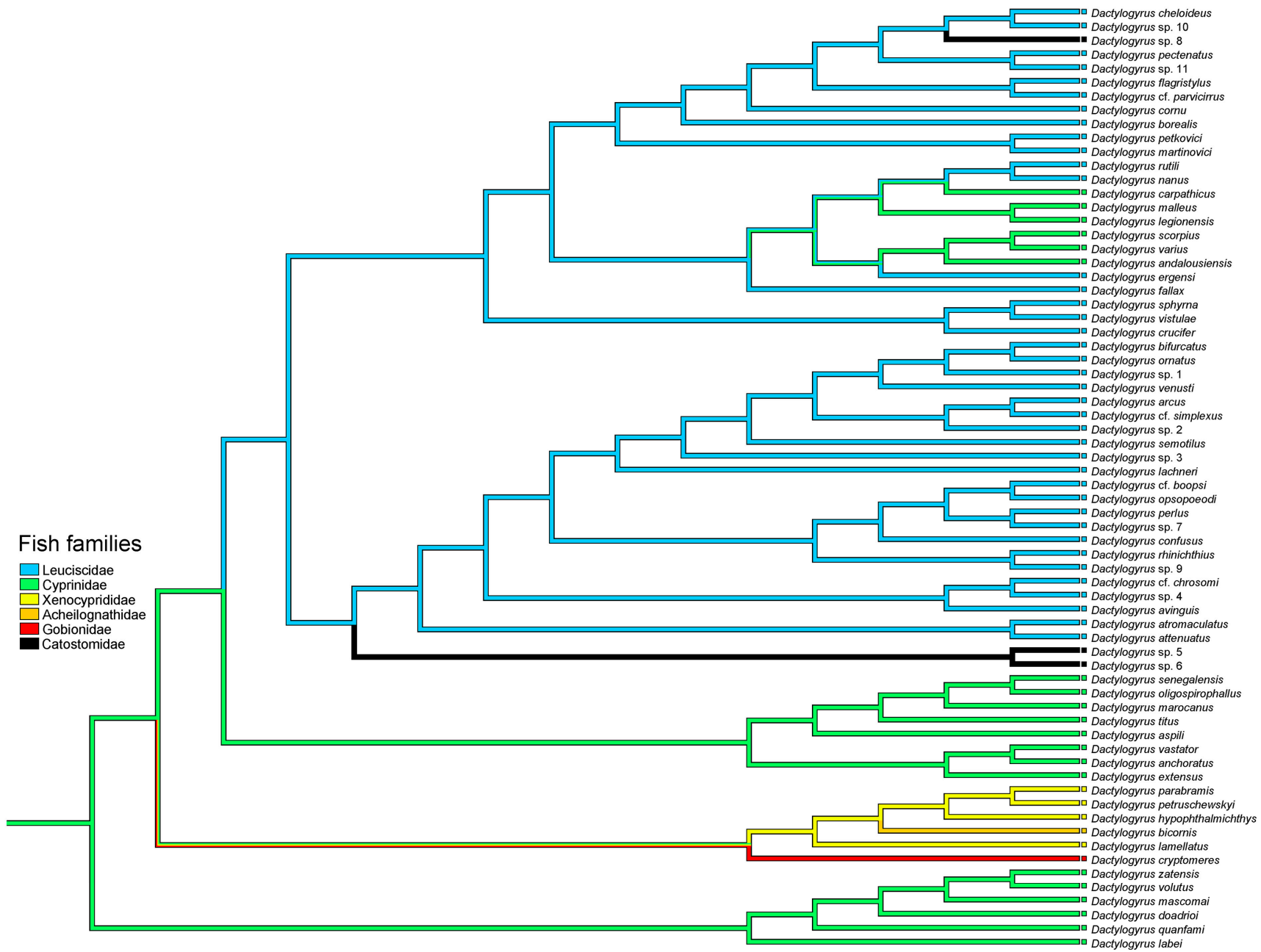

Figure 4. Mapping of fish lineages (of the cyprinoid families defined by Schönhuth et al. (2018) and Catostomidae)) onto the BI reconstruction of Dactylogyrus phylogeny.

Dactylogyrus species parasitizing Catostomidae have multiple origins. Two sister species parasitizing Hypentelium nigricans shared a common origin with Dactylogyrus spp. parasitizing fish of the CCP clade (Plagopterinae represented by S. atromaculatus in our data), whilst Dactylogyrus sp. 8 shared a common origin with two sister species parasitizing Rhinichthys (Pogonichthyinae). A host switch by D. cf. atromaculatus from S. atromaculatus (Plagopterinae) to P. notatus (Pogonichthyinae) was also revealed. 


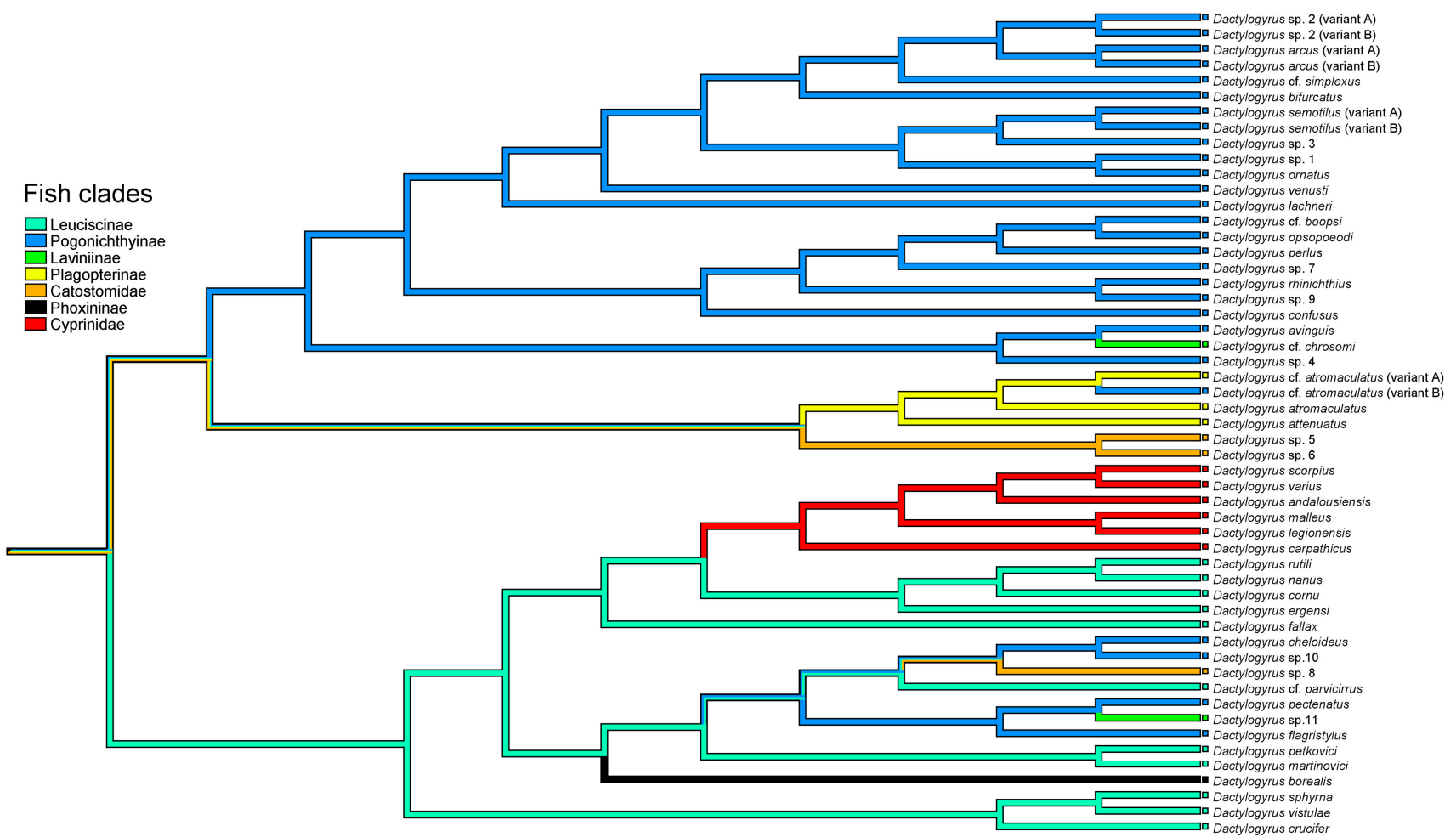

Figure 5. Mapping of fish clades (Cyprinidae, Catostomidae and the subfamilies of Leuciscidae) onto the BI phylogenetic reconstruction of Dactylogyrus of lineage IV. Clades of Leuciscidae (defined by Schönhuth et al. (2018)) are represented by Pogonichthyinae (NA clade), Leuciscinae (OW clade), Laviniinae (WC clade), Plagopterinae (CCP clade) and Phoxininae (PHX clade).

\section{Discussion}

The present study was focused on monogeneans of the genus Dactylogyrus restricted almost exclusively to cyprinoid fish species, with a view to using host-specific parasites as a potential tool for inferring biogeographical contacts between freshwater fish with fragmented distributions. Here, we investigated the phylogenetic position and origin of Nearctic Dactylogyrus spp. parasitizing Leuciscidae that also switched to a few species of Catostomidae in the North American continent. We expected the phylogenetic relationships among host-specific Dactylogyrus to reflect the historical relationships and contacts hypothesized between Nearctic and Palearctic cyprinoids [21,22].

Our phylogenetic reconstructions revealed that Dactylogyrus species parasitizing Leuciscidae in North America belong to two clades. The first clade of Nearctic Dactylogyrus species (i.e., North American Dactylogyrus clade 1 well supported on the basis of phylogenetic analyses using the concatenated data of 18SrDNA, 28SrDNA and ITS1) was restricted to north-eastern parts of the USA (Wisconsin and New York) and was nested within Dactylogyrus parasitizing European Leuciscidae and North-West African Cyprinidae. This finding indicates the European origin of North American Dactylogyrus clade 1, which is consistent with phylogenetic and biogeographical studies indicating that Leuciscidae originated in Cretaceous Europe [22]. The second clade (North American Dactylogyrus 2) included exclusively Nearctic Dactylogyrus spp. from Leuciscidae in north-eastern (Wisconsin and New York) and southern parts of the USA (Mississippi and Arkansas).

Recent phylogenetic analyses of Leuciscidae have indicated the independent evolution of leuciscin and phoxinin clades [21,22]. Imoto et al. [22] showed that the Far East Asian phoxinin (FEA) clade is closer to the North American phoxinin clade (Western clade, WC) than the European leuciscin clade (Old Word clade, OW) and proposed that phoxinins dispersed from North America to the Far East across the Bering Land Bridge in the Late Cretaceous or Paleocene [54,55]. Schönhuth et al. [21] included all clades of Holarctic Leuciscidae in their phylogenetic reconstruction and showed that Palearctic and Nearc- 
tic Leuciscidae do not form separate monophyletic groups. In contrast to the study by Imoto et al. [22], they indicated that the FEA clade, i.e., Pseudaspininae, occupied the basal position to all other clades of Leuciscidae, and that the WC clade, i.e., Laviniinae, occupied a sister position to the other Nearctic clades (i.e., the NA clade representing Pogonichthyinae and the $\mathrm{CCP}$ clade representing Plagopterinae) and to Palearctic leuciscids (the OW clade, i.e., Leuciscinae, which includes also a single Nearctic species N. crysoleucas and the Eurasian Phoxinus (PHX) clade, i.e., Phoxininae).

However, the phylogenetic position of $D$. borealis, specific to P. phoxinus, a single representative of the PHX clade in our study, does not reflect the phylogenetic relationships among clades of Leuciscidae proposed by Imoto et al. [22] or Schönhuth et al. [21], as the phylogenetic position of $D$. borealis was not supported in our phylogenetic reconstruction (according to the host-parasite database, https:/ / www.nhm.ac.uk/research-curation/ scientific-resources/taxonomy-systematics/host-parasites/database/search.jsp (accessed on 20 August 2021), D. borealis is even documented in some representatives of the FEA clade). Concerning the two Dactylogyrus species parasitizing C. neogaeus, a single representative of the WC clade in our study, their phylogenetic positions did not follow the phylogenetic position of host species in the molecular phylogenetic reconstruction of Leuciscidae, i.e., the basal position of Laviniinae to other Nearctic and Palearctic clades.

On the basis of the mapping of fish geographical distribution onto the reconstruction of parasite phylogeny, different origins of the two Nearctic Dactylogyrus clades were supported, i.e., a European origin for North American clade 1 and likely an East Asian origin for North American clade 2. Surprisingly, four leuciscid species (from a total of 10 leuciscid species parasitized by at least two Dactylogyrus species) harbored Dactylogyrus species from the two divergent North American clades with different origins, which is in contrast to the pattern of Dactylogyrus speciation previously demonstrated for European Dactylogyrus [18], i.e., intrahost speciation.

This seems to indicate that even some Nearctic leuciscid species were colonized independently by Dactylogyrus of different origins. The ancestor of North American clade 1 may have originated during the period of historical connections between Europe and North America, as indicated also for the historical dispersion of leuciscid fish. Two major North Atlantic land bridges were suggested to play a role in such connections, with the Thulean Bridge as the most important route for the exchange of biota between Europe and North America in the Early Tertiary. This land bridge connected southern Europe to eastern North America and was closed in the Early Eocene [56,57]. The second potential but less important trans-Atlantic connection for biota exchange was the northern De Greer Bridge between Scandinavia and eastern North America, which persisted until the Late Eocene. However, to clearly resolve the origin of the clade of North American Dactylogyrus 2 parasitizing Leuciscidae, we suggest that the additional sampling of Asian representatives of Dactylogyrus parasitizing Leuciscidae is necessary for future phylogenetic studies, i.e., particularly Dactylogyrus species parasitizing Pseudoaspininae (FEA clade).

It is generally hypothesized that cyprinoids originated in Oriental Asia, i.e., South and Southeast Asia $[58,59]$. Fossil records in Asia and phylogenetic analyses indicating that the FEA clade is a sister to all remaining clades of European and North American leuciscids support the hypothesis that ancestral Asian leuciscids dispersed in Eurasia and also colonized North America via the Bering Land Bridge, when the sea level decreased during the mid-Oligocene [21,60]. Therefore, an Asian origin for the clade of North American Dactylogyrus 2 appears to be a highly plausible scenario. This hypothesis may also be supported by the mapping of fish families onto the phylogenetic reconstruction of Dactylogyrus spp. in the present study, where Cyprinidae, which are of Asian origin, were shown as an early and potentially ancestral host group for Dactylogyrus spp., and Leuciscidae represented more recently evolved host groups for Dactylogyrus. A similar finding was revealed from previous reconstructions of Dactylogyrus phylogeny $[15,18]$.

Different clades of Leuciscidae are distributed in Europe and North America. In Europe, two clades of Leuciscidae are represented-namely, the highly diverse Leuciscinae 
and the species poor Phoxininae [21], whilst in the Nearctic region, four clades of Leuciscidae are present-Laviniinae; Plagopterinae; Pogonichthiynae, which is the most diverse; and a single representative of Leuciscinae (see above). The mapping of leuciscid clades onto the phylogenetic reconstruction of Dactylogyrus indicates that ancestral European Dactylogyrus parasitizing Leuciscinae likely switched to colonize representatives of the most diverse Nearctic clade of Leuciscidae, i.e., the NA clade-Pogonichthyinae.

A single Dactylogyrus species (D. cf. parvicirrus) identified on N. crysoleucas, the only Nearctic representative of Leuciscinae, was nested within the clade of North American Dactylogyrus 1, which has a European origin. The divergence between N. crysoleucas and other European leuciscin species was estimated to 37.1 Mya [22], when the land bridge between two continents was still present. Even though the mapping of leuciscid clades onto Dactylogyrus lineage IV revealed an unclear origin for Dactylogyrus host-specific to $N$. crysoleucas, it seems that $N$. crysoleucas likely lost its original host-specific gill monogeneans during its colonization of North America and was secondarily colonized by Dactylogyrus in North America (likely from Pogonichthyinae).

The mapping of leuciscid clades onto the Dactylogyrus phylogeny showed that highly diversified Pogonichthyinae is an evolutionarily old host group for Nearctic Dactylogyrus. Within North America clade 1, Dactylogyrus of Pogonichthyinae likely secondarily colonized N. crysoleucas, Laviniinae (represented by C. neogaeus in our analyses) and even a representative of Catostomidae (Catostomus commersonii). Within the clade of North American Dactylogyrus 2, Dactylogyrus of Pogonichthyinae likely secondarily colonized Plagopterinae (represented by the widely distributed S. atromaculatus in our analyses), Laviniinae (C. neogaeus) and even Catostomidae (H. nigricans). Considering the enormous diversity of Nearctic leuciscids and our limited knowledge on the potential diversity of their host-specific Dactylogyrus species (see [20]), this mapping-even if performed with a limited number of Dactylogyrus species-indicated that the two independent colonisations by Dactylogyrus from highly diversified Pogonichthyinae to Laviniinae likely occurred because of the sympatric distributions of North American leuciscids of both clades.

Dactylogyrus species are primarily parasites of cyprinoid fish (previously classified as cyprinids), i.e., $95 \%$ of species of Dactylogyrus are restricted to cyprinoid species. However, some Dactylogyrus species have also been reported on non-cyprinoid fish [17], including Catostomidae. Catostomidae are almost exclusively native to North America. Catostomus catostomus is the only extant trans-Pacific species [61], i.e., this species exhibits a disjunct distribution in the Nearctic and Palearctic (Siberia) regions, and Myxocyprinus asiaticus is the only catostomid species endemic to Eurasia and in eastern China [62].

Up to now, nine species of Dactylogyrus have been described from eight catostomid species of three genera in North America (Moxostoma, Hypentelium and Thoburnia, all representatives of the subfamily Catostominae) [20]. Our phylogenetic analyses included only three Dactylogyrus species collected on two catostomine species, H. nigricans and C. commersonii and revealed two independent colonization events involving Dactylogyrus on catostomine fish of North America. Without molecular calibration, it is impossible to estimate whether these colonization events represent deeper historical or more contemporary host switches resulting from the sympatric distribution of leuciscids and catostomids. However, catostomines diverged in the Oligocene (Catostomini and Erimyzonini) and in the early Miocene (Moxostomatini and Thoburniini), and the genus Catostomus diversified from 17.65 Ma ago in the early-mid Miocene [63]. The arrival of cyprinoids in North America during the Oligocene is hypothesized on the basis of fossil records of North American teleost fishes [60,64], i.e., in the period when some genera of catostomines were already present in North America.

In our phylogenetic reconstruction, one colonization of Catostomidae by Dactylogyrus was documented within the clade of North American Dactylogyrus 2. Two sister species of Dactylogyrus found on H. nigricans formed a monophyletic group with Dactylogyrus species parasitizing S. atromaculatus (a representative of North American Plagopterinae). We could hypothesize a host switch by Dactylogyrus from S. atromaculatus to H. nigricans, which may 
be supported by the fact that $S$. atromaculatus is one of the most common fish species in eastern North America and also widely introduced across North America [65].

The northern $\mathrm{H}$. nigricans is native to southern Canada and the eastern and southern United States. It lives in the rivers of the Mississippi Basin, its range extending from Oklahoma and Alabama northward to Minnesota [66]. The sympatric distribution of both species was also documented in our study. The other host switch by Dactylogyrus from Leuciscidae to Catostomidae was revealed within North American clade 1. Dactylogyrus sp. 8 parasitizing C. commersonii formed a well-supported clade with two Dactylogyrus species parasitizing species of Rhinichthys (representatives of the highly diversified Pogonichthyinae), which clearly provides further evidence of another host switch by Dactylogyrus to catostomids on the North American continent.

\section{Conclusions}

Host-specific monogeneans of the genus Dactylogyrus parasitizing Nearctic cypriniform fish (including Leuciscidae and a few species of Catostomidae) formed two independent clades with different origins likely associated with different historical routes of cyprinoid dispersion to the North American continent. The relationships among Dactylogyrus parasitizing different leuciscid clades in North America and Europe did not reflect the phylogenetic relationships of their leuciscid hosts.

However, the phylogenetic reconstruction of Dactylogyrus reflects the biogeographical contacts of cyprinoids across continents and even more contemporary contacts among leuciscids in the Nearctic region following the separation of the continents. We highlight the usefulness of host-specific monogeneans as a tool for studying the biogeographical contacts of hosts with fragmented/disjunct distributions.

Author Contributions: Conceptualization, A.Š.; Methodology, A.Š., E. ̌̌., A.C. and M.S.; Analyses, A.Š., E.̌̌. and M.S.; Investigation, A.Š., E.Ǩ., A.C. and M.S.; Writing-Original Draft Preparation, A.Š.; Funding Acquisition, A.Š. All authors have read and agreed to the published version of the manuscript.

Funding: This study was funded by the Ministry of Education, Youth and Sports of the Czech Republic, project no. LTAUSA18010.

Institutional Review Board Statement: Fieldwork was carried out with the approval of the competent local authorities (provided to US partners) and was approved by the Animal Care and Use Committee of the Faculty of Science, Masaryk University (Czech Republic).

Informed Consent Statement: Not applicable.

Data Availability Statement: Parasite voucher material is available at the Parasitology Laboratory, Department of Botany and Zoology, Faculty of Science, Masaryk University. DNA sequence data are available in the GenBank Nucleotide Database under accession numbers OM108517-OM108588.

Acknowledgments: We are grateful to our US colleagues Chris T. McAllister (Science and Mathematics Division, Eastern Oklahoma State College, Idabel, Oklahoma), Stephen S. Curran and Robin M. Overstreet (Gulf Coast Research Laboratory, Ocean Springs, Mississippi) and Florian B. Reyda (State University of New York, Oneonta College, Oneonta, New York Tomas) for fish sampling and fish determination; and to our Czech colleagues Kateřina Francová (Department of Botany and Zoology, Faculty of Science, Masaryk University), Tomáš Scholz and Roman Kuchta (Institute of Parasitology, Biology Centre of the Czech Academy of Science) for fish dissection and parasite collection. A.C. acknowledges support from St. Norbert College. We kindly thank Matthew Nicholls for the English revision of the final draft.

Conflicts of Interest: The authors declare that they have no competing interests.

\section{References}

1. Brooks, D.R.; Hoberg, E.P. Triage for the biosphere: The need and rationale for taxonomic inventories and phylogenetic studies and parasites. Comp. Parasitol. 2000, 67, 1-25. 
2. Hoberg, E.P.; Klassen, G.J. Revealing the faunal tapestry: Co-evolution and historical biogeography of hosts and parasites in marine systems. Parasitology 2002, 124, S3-S22. [CrossRef] [PubMed]

3. Brooks, D.R.; McLennan, D.A. Parascript: Parasites and the Language of Evolution; Smithsonian Institution Press: Washington, DC, USA, 1993.

4. Brooks, D.R.; McLennan, D.A. The Nature of Diversity: An Evolutionary Voyage of Discovery; University of Chicago Press: Chicago, IL, USA, 2002.

5. Řehulková, E.; Seifertová, M.; Přikrylová, I.; Francová, K. Monogenea. In A Guide to the Parasites of African Freshwater Fishes; Scholz, T., Vanhove, M.P.M., Smit, N., Jayasundera, Z., Gelnar, M., Eds.; AbcTaxa: Brussels, Belgium, 2018; pp. 185-243.

6. Rohde, K. A non-competitive mechanism responsible for restricting niches. Zool. Anz. 1977, 199, $164-172$.

7. Rohde, K. Simple ecological systems, simple solution to complex problems? Evol. Theor. 1989, 8, 305-350.

8. Euzet, L.; Combes, C. The selection of habitats among the monogenea. Int. J. Parasitol. 1998, 28, 1645-1652. [CrossRef]

9. Šimková, A.; Ondračková, M.; Gelnar, M.; Morand, S. Morphology and coexistence of congeneric ectoparasite species: Reinforcement of reproductive isolation? Biol. J. Linn. Soc. 2002, 76, 125-135. [CrossRef]

10. Šimková, A.; Verneau, O.; Gelnar, M.; Morand, S. Specificity and specialization of congeneric monogeneans parasitizing cyprinid fish. Evolution 2006, 60, 1023-1037. [CrossRef]

11. Pariselle, A.; Boeger, W.A.; Snoeks, J.; Bilong Bilong, C.F.; Morand, S.; Vanhove, M.P.M. The monogenean parasite fauna of cichlids: A potential tool for host biogeography. Int. J. Evol. Biol. 2011, 2011, 471480. [CrossRef]

12. Benovics, M.; Desdevises, Y.; Vukić, J.; Šanda, R.; Šimková, A. The phylogenetic relationships and species richness of host-specific Dactylogyrus parasites shaped by the biogeography of Balkan cyprinids. Sci. Rep. 2018, 8, 13006. [CrossRef]

13. Benovics, M.; Vukić, J.; Šanda, R.; Rahmouni, I.; Šimková, A. Disentangling the evolutionary history of peri-Mediterranean cyprinids using host-specific gill monogeneans. Int. J. Parasitol. 2020, 50, 969-984. [CrossRef]

14. Benovics, M.; Desdevises, Y.; Šanda, R.; Vukić, J.; Scheifler, M.; Doadrio, I.; Sousa-Santos, C.; Šimková, A. High diversity of fish ectoparasitic monogeneans (Dactylogyrus) in the Iberian Peninsula: A case of adaptive radiation? Parasitology 2000, 147, 418-430. [CrossRef]

15. Šimková, A.; Benovics, M.; Rahmouni, I.; Vukić, J. Host-specific Dactylogyrus parasites revealing new insights on the historical biogeography of Northwest African and Iberian cyprinid fish. Parasit. Vectors 2017, 10, 589. [CrossRef]

16. Winfield, I.J.; Nelson, J.S. Cyprinid Fishes: Systematics, Biology and Exploitation; Fish and Fisheries Series No. 3; Chapman and Hall: London, UK, 1991.

17. Gibson, D.I.; Timofeeva, T.A.; Gerasev, P.I. A catalogue of the nominal species of the monogenean genus Dactylogyrus Diesing, 1850 and their host genera. Syst. Parasitol. 1996, 35, 3-48. [CrossRef]

18. Šimková, A.; Morand, S.; Jobet, E.; Gelnar, M.; Verneau, O. Molecular phylogeny of congeneric monogenean parasites (Dactylogyrus): A case of intrahost speciation. Evolution 2004, 58, 1001-1018. [CrossRef]

19. Benovics, M.; Kičinjaová, M.L.; Šimková, A. The phylogenetic position of the enigmatic Balkan Aulopyge huegelii (Teleostei: Cyprinidae) from the perspective of host specific Dactylogyrus parasites (Monogenea), with a description of Dactylogyrus omenti $\mathrm{n}$. sp. Parasit. Vectors 2017, 10, 547. [CrossRef]

20. Kuchta, R.; Řehulková, E.; Francová, K.; Scholz, T.; Morand, S.; Šimková, A. Diversity of monogeneans and tapeworms in cypriniform fishes across two continents. Int. J. Parasitol. 2020, 50, 771-786. [CrossRef]

21. Schönhuth, S.; Vukić, J.; Šanda, R.; Yang, L.; Mayden, R.L. Phylogenetic relationships and classification of the Holarctic family Leuciscidae (Cypriniformes: Cyprinoidei). Mol. Phyl. Evol. 2018, 127, 781-799. [CrossRef]

22. Imoto, J.M.; Saitoh, K.; Sasaki, T.; Yonezawa, T.; Adachi, J.; Kartavtsev, Y.P.; Miya, M.; Nishida, M.; Hanzawa, N. Phylogeny and biogeography of highly diverged freshwater fish species (Leuciscinae, Cyprinidae, Teleostei) inferred from mitochondrial genome analysis. Gene 2013, 514, 112-124. [CrossRef]

23. Banarescu, P. Some reconsiderations of the zoogeography of the euro-Mediterranean freshwater fish fauna. Rev. Romane Biol. Zool. 1973, 8, 257-264.

24. Doadrio, I. Phylogenetic relationships and classification of western Palearctic species of the genus Barbus (Osteichthyes, Cyprinidae). Aquat. Living Resour. 1990, 3, 265-282. [CrossRef]

25. Benovics, M.; Nejat, F.; Abdoli, A.; Šimková, A. Molecular and morphological phylogeny of host-specific Dactylogyrus parasites (Monogenea) sheds new light on the puzzling Middle Eastern origin of European and African lineages. Parasit. Vectors 2021, 14, 372. [CrossRef]

26. Mizelle, J.D. Ectoparasites of the blunt-nosed minnow (Hyborhynchus notatus). Am. Midl. Nat. 1937, 18, 612-621. [CrossRef]

27. Mizelle, J.D. New species of monogenetic flukes from Illinois fishes. Am. Midl. Nat. 1938, 19, 465-470. [CrossRef]

28. Mueller, J.F. Additional species of North American Gyrodactyloidea (Trematoda). Am. Midl. Nat. 1938, 19, 220-235. [CrossRef]

29. Seamster, A. Two new Dactylogyridae (Trematoda: Monogenea) from the golden shiner. J. Parasitol. 1948, 34, 111-113. [CrossRef]

30. Mizelle, J.D.; Klucka, A.R. Studies on monogenetic trematodes XIV. Dactylogyridae from Wisconsin fishes. Am. Midl. Nat. 1953, 49, 720-733. [CrossRef]

31. Monaco, L.H.; Mizelle, J.D. Studies on monogenetic trematodes XVII. The genus Dactylogyrus. Am. Midl. Nat. 1955, 53, 455-477. [CrossRef]

32. Wood, R.A.; Mizelle, J.D. Studies on monogenetic trematodes. XXI. North American Gyrodactylinae, Dactylogyrinae and a new host record for Urocleidus dispar (Mueller, 1936). Am. Midl. Nat. 1957, 57, 183-202. [CrossRef] 
33. Rogers, W.A. Studies on Dactylogyrinae (Monogenea) with descriptions of 24 new species of Dactylogyrus, 5 new species of Pellucidhaptor, and the proposal of Aplodiscus gen. n. J. Parasitol. 1967, 53, 501-524. [CrossRef]

34. Chien, S.-M. Dactylogyrids from North American cyprinids of the genus Nocomis. The reciprocus species group. J. Parasitol. 1971, 57, 1211-1214. [CrossRef]

35. Chien, S.-M. Dactylogyrids from North American cyprinids of the genus Nocomis: The bellicus group. J. Parasitol. 1974, 60, 585-594. [CrossRef] [PubMed]

36. Chien, S.-M. Dactylogyrids from North American cyprinids of the genus Nocomis: The Limulus and the Mollis groups. J. Parasitol. 1974, 60, 773-776. [CrossRef] [PubMed]

37. Hanek, G.; Molnár, K.; Fernando, C.H. New and previously known Dactylogyrus spp. from southern Ontario fishes. J. Parasitol. 1975, 61, 421-426. [CrossRef]

38. Mayes, M.A. New species of Gyrodactylus and Dactylogyrus (Trematoda: Monogenea) from fishes of Nebraska. J. Parasitol. 1977, 63, 805-809. [CrossRef] [PubMed]

39. Cloutman, D.G. Dactylogyrus boopsi sp. n. (Monogenea: Dactylogyridae) from the bigeye shiner, Notropis boops Gilbert (Pisces: Cyprinidae). J. Helminthol. Soc. Washingt. 1994, 61, 219-220.

40. Cloutman, D.G.; Rogers, W.A. Determination of the Dactylogyrus banghami complex (Monogenea: Dactylogyridae) from North American Gulf of Mexico coastal drainages with descriptions of three new species. Comp. Parasitol. 2005, 72, 10-16. [CrossRef]

41. Sinnappah, N.D.; Lim, L.H.; Rohde, K.; Tinsley, R.; Combes, C.; Verneau, O. A Paedomorphic parasite associated with a neotenic amphibian host: Phylogenetic evidence suggests a revised systematic position for Sphyranuridae within anuran and turtle Polystomatoineans. Mol. Phyl. Evol. 2001, 18, 189-201. [CrossRef]

42. Hassouna, N.; Michot, B.; Bachellerie, J.P. The complete nucleotide sequence of mouse $28 \mathrm{~S}$ rRNA gene. Implications for the process of size increase of the large subunit rRNA in higher eukaryotes. Nucleic Acids Res. 1984, 12, 3563-3583. [CrossRef]

43. Kumar, S.; Stecher, G.; Li, M.; Knyaz, C.; Tamura, K. MEGA X: Molecular Evolutionary Genetics Analysis across computing platforms. Mol. Biol. Evol. 2018, 35, 1547-1549. [CrossRef]

44. Katoh, K.; Standley, D.M. MAFFT multiple sequence alignment software version 7: Improvements in performance and usability. Mol. Biol. Evol. 2013, 30, 772-780. [CrossRef]

45. Talavera, G.; Castresana, J. Improvement of phylogenies after removing divergent and ambiguously aligned blocks from protein sequence alignments. Syst. Biol. 2007, 56, 564-577. [CrossRef]

46. Darriba, D.; Taboada, G.L.; Doallo, R.; Posada, D. jModelTest 2: More models, new heuristics and parallel computing. Nat. Methods 2012, 9, 772. [CrossRef]

47. Nguyen, L.T.; Schmidt, H.A.; Von Haeseler, A.; Minh, B.Q. IQ-TREE: A fast and effective stochastic algorithm for estimating maximum-likelihood phylogenies. Mol. Biol. Evol. 2015, 32, 268-274. [CrossRef]

48. Trifinopoulos, J.; Nguyen, L.T.; von Haeseler, A.; Minh, B.Q. W-IQ-TREE: A fast online phylogenetic tool for maximum likelihood analysis. Nucleic Acids Res. 2016, 44, W232-W235. [CrossRef]

49. Minh, B.Q.; Nguyen, M.A.T.; von Haeseler, A. Ultrafast approximation for phylogenetic bootstrap. Mol. Biol. Evol. 2013, 30, 1188-1195. [CrossRef]

50. Ronquist, F.; Teslenko, M.; Van Der Mark, P.; Ayres, D.L.; Darling, A.; Höhna, S.; Larget, B.; Liu, L.; Suchard, M.A.; Huelsenbeck, J.P. MrBayes 3.2: Efficient Bayesian phylogenetic inference and model choice across a large model space. Syst. Biol. 2012, 61, 539-542. [CrossRef]

51. Rambaut, A.; Drummond, A.J.; Xie, D.; Baele, G.; Suchard, M.A. Posterior summarization in Bayesian phylogenetics using Tracer 1.7. Syst. Biol. 2018, 67, 901. [CrossRef]

52. Rambaut, A. FigTree, Version 1.4.4; 2018. Available online: http://tree.bio.ed.ac.uk/software/figtree/ (accessed on 15 June 2021 ).

53. Maddison, W.; Maddison, D. Mesquite 3.2. A Modular System for Evolutionary Analysis. 2019. Available online: http: / / mesquiteproject.org (accessed on 15 June 2021).

54. Marincovich, L.; Gladenkov, A.Y. Evidence for an early opening of the Bering Strait. Nature 1999, 397, 149-151. [CrossRef]

55. Marincovich, L.; Gladenkov, A.Y. New evidence for the age of Bering Strait. Quatr. Sci. Rev. 2001, 20, 20,329-335. [CrossRef]

56. McKenna, M.C. Cenozoic paleontology of North Atlantic land bridges. In Structure and Development of the Greenland-Scotland Bridge: New Concepts and Methods; Bott, M.H.P., Saxov, S., Talwani, M., Thiede, J., Eds.; Plenum: New York, NY, USA, 1983.

57. Tiffney, B.H. The Eocene North Atlantic land bridge: Its importance in tertiary and modern phytogeography of the northern hemisphere. J. Arnold Arbor. 1985, 66, 243-273. [CrossRef]

58. Banarescu, P. The zoogeographical position of the East Asian freshwater fish fauna. Rev. Roumanie Biol. Ser. Zool. 1972, 17, 315-323.

59. Briggs, J.C. Ostariophysan zoogeography: An alternative hypothesis. Copeia 1979, 1, 111-118. [CrossRef]

60. Cavender, T.M. The fossil record of the Cyprinidae. In Cyprinid Fishes, Systematics, Biology and Exploitation; Winfield, I.J., Nelson, J.S., Eds.; Chapman and Hall: London, UK, 1991; pp. 34-54.

61. Harris, P.M.; Hubbard, G.; Sandel, M. Catostomidae: Suckers. In Freshwater Fishes of North America: Volume 1: Petromyzontidae to Catostomidae; Warren, M.L., Burr, B.M., Eds.; Johns Hopkins University Press: Baltimore, MD, USA, 2014; pp. 451-502.

62. Smith, G.R. Phylogeny and biogeography of the Catostomidae, freshwater fishes of North America and Asia. In Systematics, Historical Ecology, and North American Freshwater Fishes; Mayden, R.L., Ed.; Stanford University Press: Stanford, CA, USA, 1992; pp. 778-813. 
63. Bagley, J.C.; Mayden, R.L.; Harris, P.M. Phylogeny and divergence times of suckers (Cypriniformes: Catostomidae) inferred from Bayesian total evidence analyses of molecules, morphology, and fossils. Peer J. 2018, 6, e5168. [CrossRef]

64. Cavender, T. Review of the fossil history of North American freshwater fishes. In The Zoogeography of North American Freshwater Fishes; Hocutt, C.H., Wiley, E.O., Eds.; John Wiley and Sons: New York, NY, USA, 1986; pp. 699-724.

65. Froese, R.; Pauly, D. FishBase. World Wide Web Electronic Publication. Available online: www.fishbase.org (accessed on 15 June 2021).

66. Raney, E.C.; Lachner, E.A. Age, growth, and habits of the hog sucker, Hypentelium nigricans (LeSueur). N. Y. Am. Midl. Nat. 1946, 36, 76-86. [CrossRef] 